\title{
Effective interactions between soft-repulsive colloids: Experiments, theory, and simulations
}

\author{
Priti S. Mohanty, ${ }^{1, a)}$ Divya Paloli, ${ }^{1,2}$ Jérôme J. Crassous, ${ }^{1}$ Emanuela Zaccarelli, ${ }^{3, a)}$ \\ and Peter Schurtenberger ${ }^{1}$ \\ ${ }^{1}$ Division of Physical Chemistry, Lund University, Lund, Sweden \\ ${ }^{2}$ Department of Chemistry, University of Fribourg, Fribourg, Switzerland \\ ${ }^{3}$ CNR-ISC and Dipartimento di Fisica, Sapienza Università di Roma, P.le A. Moro 2, I-00185 Rome, Italy
}

\begin{abstract}
We describe a combined experimental, theoretical, and simulation study of the structural correlations between cross-linked highly monodisperse and swollen Poly( $\mathrm{N}$-isopropylacrylamide) microgel dispersions in the fluid phase in order to obtain the effective pair-interaction potential between the microgels. The density-dependent experimental pair distribution functions $g(r)$ 's are deduced from real space studies using fluorescent confocal microscopy and compared with integral equation theory and molecular dynamics computer simulations. We use a model of Hertzian spheres that is capable to well reproduce the experimental pair distribution functions throughout the fluid phase, having fixed the particle size and the repulsive strength. Theoretically, a monodisperse system is considered whose properties are calculated within the Rogers-Young closure relation, while in the simulations the role of polydispersity is taken into account. We also discuss the various effects arising from the finite resolution of the microscope and from the noise coming from the fast Brownian motion of the particles at low densities, and compare the information content from data taken in 2D and 3D through a comparison with the corresponding simulations. Finally different potential shapes, recently adopted in studies of microgels, are also taken into account to assess which ones could also be used to describe the structure of the microgel fluid.
\end{abstract}

\section{INTRODUCTION}

Colloidal suspensions have frequently been used as convenient model systems to address various phase transitions in condensed matter physics. ${ }^{1-4}$ While early works concentrated primarily on hard sphere-like and charged (Yukawa) particles, soft colloids have recently moved into the focus of the soft matter community. Soft colloids are a class of particles where the average interparticle distance can be smaller than the particle diameter and correspondingly the effective interaction potential shows a finite repulsion at or beyond contact. ${ }^{5-7}$ In particular, cross-linked microgels such as poly $(N$-isopropylacrylamide) (PNIPAM) that can undergo reversible volume phase transitions upon variations of external stimuli such as temperature, $\mathrm{pH}$, ionic strength or hydrostatic pressure $^{8,9}$ have been employed frequently as model systems for soft colloids. ${ }^{10-17}$

The physical nature of these particles lies between that of classical hard sphere colloids and ultra-soft polymeric colloids such as star polymers. ${ }^{5,18}$ Due to their soft-repulsive character, microgels can compress or interpenetrate to a certain degree, which allows to create states with densities far above close packing, so called squeezed states, with interesting structural and dynamical properties. ${ }^{13,15-17}$ A number of previous studies on PNIPAM microgel dispersions have focused on their microstructure, and their concentration

\footnotetext{
a) Authors to whom correspondence should be addressed. Electronic addresses: priti.mohanty@fkem1.lu.se and emanuela.zaccarelli@phys. uniroma1.it
}

and temperature dependent phase behavior. ${ }^{10-17}$ However, despite all the experimental and theoretical efforts devoted to these systems, the exact nature of the interaction potential between microgels and its dependence on the particle concentration as a vital ingredient for any attempt to theoretically model and understand these systems still needs to be assessed.

Soft-repulsive colloids have been often modeled by different forms of an effective interaction pair potential such as an inverse power law, ${ }^{6}$ a Gaussian core, ${ }^{19}$ a Hertzian, ${ }^{20}$ or a harmonic potential. ${ }^{21}$ All these different potentials theoretically result in a rich and complex phase behavior, which, however, has not been experimentally asserted yet. The interaction potential between microgels has also been addressed in a number of experimental studies. ${ }^{15-17,22}$ However, a major problem in an experimental study of the interaction potential between microgels arises from the intrinsic softness of the particles in their fully swollen state as this could also lead to a concentration dependence of the particle size and thus of the volume fraction and potential.

Various experimental approaches such as small-angle neutron scattering (SANS), ${ }^{23}$ confocal laser scanning microscopy (CLSM), ${ }^{16,17,24}$ or rheological investigations ${ }^{10,25}$ have been used in the attempts to shed light on the effective interaction potential between microgels. Examples are an evaluation of structural data using CLSM at low effective volume fractions $\phi_{\mathrm{eff}},{ }^{17,24}$ or the analysis of the $\phi_{\mathrm{eff}}{ }^{-}$ dependence of the shear modulus $\left(G_{p}\right)$ in highly concentrated microgel suspensions based on rheological studies ${ }^{10}$ and 
diffusing wave spectroscopy, ${ }^{15}$ respectively. However, until recently we lacked a systematic study of the interactions between microgel particles over a large range of volume fractions, i.e., in the entire fluid regime.

This has been addressed in the context of a recent study of fluid-solid transitions in microgel suspensions, where we have shown that uncharged and densely cross-linked microgel particles in the swollen state interact via a Hertzian potential in the entire fluid regime below freezing. This was achieved through a quantitative comparison of the experimental pair correlation functions $g(r)$ 's obtained using CLSM with the results from computer simulations. ${ }^{17}$ As a follow-up of this study, we now provide a detailed account of the experimental determination of $g(r)$ in the fluid regime, and describe the parallel computer simulations and theoretical calculations required in an attempt to subject the different interaction potential forms to a critical test. Moreover, we also discuss the influence of various experimental artifacts such as the finite resolution of the microscope and projection errors that affect the experimental 2D and 3D $g(r)$ through a comparison with the corresponding simulations.

\section{MATERIALS AND METHODS}

\section{A. Synthesis and particle characterization}

We use fluorescently labeled PNIPAM microgels and CLSM to study the structural ordering at various packing fractions in the fluid phase. Different values of $\phi_{\text {eff }}$ are achieved by varying the weight concentration or number density at constant temperature $T$. Frequently, the temperatureresponsiveness of the microgels is used to vary $\phi_{\text {eff }}$ through a variation of $T$. We have however chosen to change the number density rather than vary $T$ in order to ensure that we avoid a possible alteration of the interaction potential in the course of a temperature-induced change in the degree of swelling.

PNIPAM microgel particles were synthesized by free-radical precipitation polymerization of the $\mathrm{N}$ isopropylacrylamide (NIPAM) monomers in presence of the $N, N^{\prime}$-methylene-bis-acrylamide (BIS) cross-linker (5 mol\%) and fluorescently labeled with copolymerized methacryloxyethyl thiocarbamoyl rhodamine B (MRB) as described in more details previously. ${ }^{17}$

The swelling behavior of the particles was studied by means of dynamic light scattering using a goniometer based instrument that implements a modulated 3D cross correlation method (LS instruments, Switzerland) at a wavelength of $\lambda=632.8 \mathrm{~nm}$. The experiments were carried out in noninteracting highly dilute suspensions. The normalized intensity cross correlation function $g^{(2)}(q, t)$ was measured with 3D dynamic light scattering (3D DLS) in an angular range $30^{\circ} \leq \theta \leq 50^{\circ} \mathrm{C}$ every $5^{\circ}$, where $\theta$ is the scattering angle. Here, the magnitude of the corresponding scattering vectors given by $q=\frac{4 \pi n_{0}}{\lambda} \sin \left(\frac{\theta}{2}\right)$ with $n_{0}$ the refractive index of water, is sufficiently low to ensure that the measurements are not affected by the particle form factor. The field autocorrelation function, $g^{(1)}(q, t)$, was extracted using the Siegert relation. Then, $g^{(1)}(q, t)$ was analyzed using a second order cumulant analysis given by

$$
\ln g^{(1)}(q, t)=-\Gamma t+\frac{\mu_{2} t^{2}}{2},
$$

where $\Gamma$ is the average decay rate and $\mu_{2}$ is the second-order cumulant coefficient that is related to the degree of polydispersity by $\mu_{2} / \Gamma^{2}$. From $\Gamma=D_{0} q^{2}$ we have then calculated the translational free particle diffusion coefficient $D_{0}$. The hydrodynamic diameter $\sigma_{h}$ was finally derived through the StokesEinstein-Sutherland relation $D_{0}=k_{B} T /\left(3 \pi \eta \sigma_{h}\right)$, where $k_{B}$ is the Boltzmann constant, $T$ is the absolute temperature, and $\eta$ is the solvent viscosity.

The measured swelling curve is shown in Fig. 1(a). With increasing temperature, the particle size decreases and a volume phase transition (VPT) occurs around $33^{\circ} \mathrm{C}$. Below the VPT the particles are highly water swollen, as water then is a good solvent for PNIPAM. Above the VPT, water now acts as a poor solvent, leading to a complete collapse of the microgels at sufficiently high temperatures, i.e., at or above $40^{\circ} \mathrm{C}$ (Fig. 1(a)). The swelling ratio at $15^{\circ} \mathrm{C}$ defined as $\left(R_{h}\left(15^{\circ} \mathrm{C}\right) / R_{h}\left(45^{\circ} \mathrm{C}\right)\right)^{3}$ is equal to 5.5 .

Small angle X-ray scattering( SAXS) experiments were performed at the cSAXS beamline of the Swiss Light Source (SLS) at the Paul Scherrer Institute, Switzerland in order to measure the form factor of the microgels in the swollen state (at $T=15.6^{\circ} \mathrm{C}$ ) at low concentration. The form-factor is fitted with a core-shell structure described by the so-called fuzzy sphere model. ${ }^{11}$ This fuzzy sphere model form factor

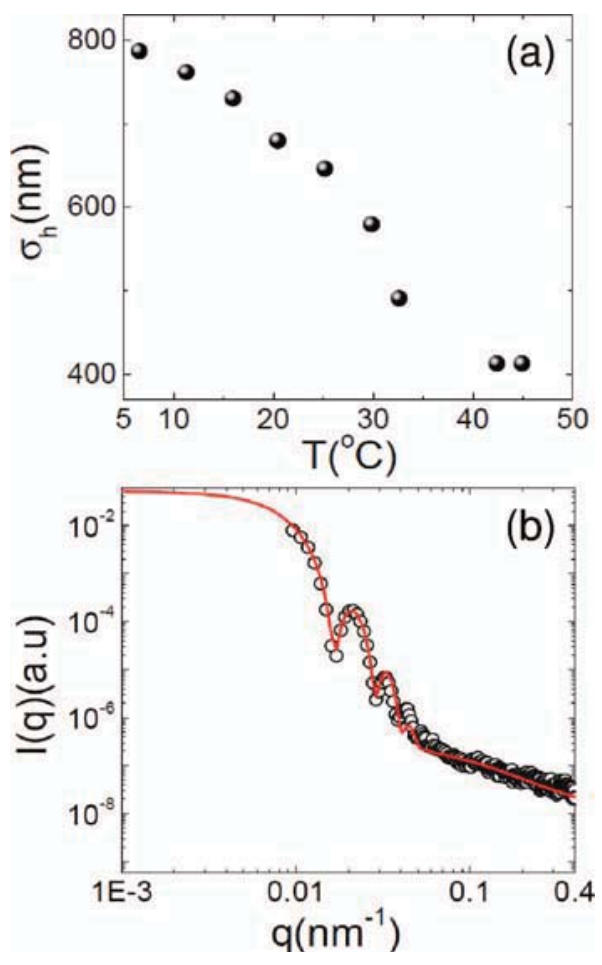

FIG. 1. (a) Average hydrodynamic diameter $\sigma_{h}$ as a function of temperature measured by DLS for a dilute microgel suspension. The volume phase transition (VPT) occurs around the lower critical solution temperature of PNIPAM at $33^{\circ} \mathrm{C}$. (b) Form-factor of microgels measured by SAXS in the swollen state at a temperature $T=15.6{ }^{\circ} \mathrm{C}$ (open black symbol). Red line is fitted curve using fuzzy sphere model. 
is given by

$$
P(q)=\left[\frac{3(\sin q R-q R \cos q R)}{(q R)^{3}} \exp \left(-\frac{\left(\sigma_{\text {surf }} q\right)^{2}}{2}\right)\right]^{2} .
$$

Equation (2) describes the scattering from a monodisperse sphere with a fuzzy outer shell whose density gradually decreases as an error function. $R$ is the half-width radius and $2 \sigma_{\text {surf }}$ is the half-width of the fuzzy shell. In order to capture polydispersity, the form factor is then integrated over the size distribution approximated by a Gaussian distribution using the appropriate intensity weighting. In addition, a Lorentzian function describing the scattering from the fluctuation of the network is added to the fuzzy sphere model function and is given by

$$
L(q)=L(0) /\left[1+(\xi q)^{2}\right]
$$

where $L(0)$ is the intensity at $q=0$ and $\xi$ accounts for the correlation length of the fluctuations and is related to the mesh size.

The fit to the corresponding form factor using a polydispersity of $4 \%$ is shown in Fig. 1(b). From the fit we obtain $R$ $=268 \mathrm{~nm}$ and $2 \sigma_{\text {surf }}=84 \mathrm{~nm}$ and thus an overall radius of $R_{\text {SAXS }}=R+2 \sigma_{\text {surf }}=352 \mathrm{~nm}$ with a mesh size of $\xi=11 \mathrm{~nm}$.

CLSM was employed to study the structural ordering at various packing fractions in the fluid phase. Real space studies were carried out at $15^{\circ} \mathrm{C}$ with an inverted CLSM (Leica DMI6000 and a SP5 tandem scanner in the resonant mode) at 50 frames/s at $512 \times 256$ with an excitation wavelength of $543 \mathrm{~nm}$ using a $63 \mathrm{x}$ immersion objective with a numerical aperture of 1.4. The CLSM is mounted in a thermostated enclosure which allows us to maintain the temperature with an accuracy of $\pm 0.2^{\circ} \mathrm{C}$. Samples for the confocal studies are prepared using rectangular capillary tubes with an inner diameter of $0.1 \times 2.0 \mathrm{~mm}$ (Vitrotubes Inc). All measurements were done more than $10 \mu \mathrm{m}\left(\sim 15 \sigma_{h}\right)$ away from the surface of the cover slide to minimize wall effects. 3D pair correlation functions $g(r)$ were calculated using the coordinates $\left(x_{j}\right.$, $y_{j}, z_{j}$ ) of the tracked particles obtained from the 3D stacks of $2 \mathrm{~d}$ images averaged over different regions of the sample. Effective volume fractions $\phi_{\text {eff }}$ are determined by first measuring the particle number density $n_{p}$ directly from extensive 3D scans with the CLSM in a concentrated mother suspension where the particles diffusion is arrested. $\phi_{\text {eff }}$ is then calculated using $\phi_{\text {eff }}=(\pi / 6) \sigma^{3} n_{p}$, where $\sigma$ is the diameter of the particle in the swollen state at $T=15^{\circ} \mathrm{C}$. We use an average diameter $\sigma=0.714 \mu \mathrm{m}$ based on the DLS and SAXS measurements.

The resolution of the CLSM for determining the center of mass of the particles is crucial for the quantitative determination of $g(r)$. We estimated this by measuring fluorescently labeled highly monodisperse PMMA particles of similar size that were fixed in optical glue to remove the additional uncertainty caused by the rapid thermal motion of the particles in the liquid phase. We find values for the error in the center coordinates of $\Delta x \approx \Delta y \approx 11 \mathrm{~nm}$, and $\Delta z \approx 23 \mathrm{~nm}$, i.e., an error of $\Delta x / \sigma \approx \Delta y / \sigma \approx 0.015$ and of $\Delta z / \sigma \approx 0.03$.
We subsequently take this into account in our comparisons with theory/simulation. These values also need to be compared to the optical resolution of the CLSM, which is about $220 \mathrm{~nm}$ in the $x-y$-plane and about $600 \mathrm{~nm}$ in the $z$-direction.

\section{B. Models, theory, and simulations}

To theoretically describe the effective pair-interaction potential between microgel particles, we first consider a Hertzian potential which describes the interactions between elastic spheres, ${ }^{20}$

$$
U_{H e r t z}(r)= \begin{cases}\epsilon_{H}(1-r / \sigma)^{5 / 2}, & r \leq \sigma \\ 0 & r>\sigma\end{cases}
$$

where $\epsilon_{H}$ is the repulsive strength of the potential and $\sigma$ is the diameter of the particles. To optimize the potential parameters in comparison with the experimental results, we first solve the Ornstein-Zernike equation to compute the radial distribution function within the Rogers-Young (RY) closure, ${ }^{26}$ which is known to provide good results for purely repulsive potentials. ${ }^{27-29}$ In addition, the RY approximation guarantees thermodynamic consistency of the results. In our analysis, the diameter value, and accordingly the packing fractions, are taken directly from the experiments so that the only parameter left to vary is the repulsion strength $\epsilon_{H}$. The use of integral equation theories allows us to produce a fast scanning of the phase diagram for several values of $\phi$ and $\epsilon_{H}$, and therefore it provides an efficient method to rapidly obtain the right potential parameters in comparison to experiments. However, RY suffers, as all integral equations, of being an approximate method, therefore we compare the theoretical results with numerical simulations, in order to verify their validity.

We perform standard molecular dynamics (MD) simulations (NVT equilibration, followed by NVE production runs) of a system composed of $N=2000$ particles in a cubic box at varying packing fractions. We also consider polydisperse spheres with average diameter $\sigma$, where the individual sizes are taken from a Gaussian distribution of standard deviation $s=0.04$. To compute the 3D radial distribution function, we average over at least 100 independent configurations for each state point. The simulations also allow us to compute the $2 \mathrm{D} g(r)$ taken from slices of the simulation box with various thicknesses. To ensure that each slice contains enough particles, we have carried out additional simulations of $N=50000$ particles for the desired state points in order to have a good estimate for the 2D $g(r)$. In this case, the box is cut along one direction and the results are averaged over different slices.

Finally, in order to mimic the uncertainty in the experimental measurements, we have also considered the effect of adding noise along one or more directions to the configurations obtained by MD simulations. ${ }^{30}$ In accordance with the poorer experimental resolution along the $z$-direction, we randomly displace one of the coordinate (e.g., z) of all particles in each configuration by a Gaussian noise taken from a distribution with standard deviation $w$ centered around unity. 
Then, we recalculate $g(r)$ for such modified configurations for different values of $w$. The effect of the noise is that of smearing out the peaks, in analogy with the effect of increasing polydispersity. Good agreement with the experimental results is found for all packing fractions using a noise of width $w=0.025$ along one direction (e.g., $z$ ) only, which reasonably correlates with the reduced resolution of the CLSM along the $z$-direction.

To complete the study we then extend the theoretical calculations to other types of potentials recently applied to describe effective microgel interactions. The simplest models that have been considered show a dependence on a single length scale $\sigma$, that is the (outer) diameter of the microgel particles, similar to the Hertzian model discussed so far. ${ }^{6,10,21}$ More sophisticated models adopt two levels of description, respectively, for the inner core and for the corona around it. ${ }^{15,22}$

With respect to the former class of potentials, some authors have for instance considered a Harmonic potential with a spring constant $2 \epsilon_{\text {harm }},{ }^{21}$

$$
U_{\text {harm }}(r)= \begin{cases}\epsilon_{\text {harm }}(1-r / \sigma)^{2}, & r \leq \sigma \\ 0 & r>\sigma,\end{cases}
$$

while others have proposed to use inverse power law pair potentials, ${ }^{6}$

$$
U_{n}(r)=\epsilon_{n}(\sigma / r)^{n},
$$

where $n$ is the power-law exponent describing the potential softness and $\epsilon_{n}$ is the associated interaction strength. Senff and Richtering, for example, found that an inverse power law pair potential with $n \approx 12$ could satisfactorily describe their rheological data. ${ }^{10}$ It is to be noted that for this class of models, the potential does not strictly go to zero at $\sigma$, and the concept of an effective diameter ${ }^{26}$ needs to be introduced ${ }^{26}$ to scale appropriately the unit length.

More recently, a brush-like model for microgel particles was introduced by Scheffold and co-workers, ${ }^{15}$ where the soft corona layer around the inner core was modeled using the Alexander-De Gennes scaling, while the inner core was assumed to be incompressible. The result is an effective harmonic potential, whose spring constant explicitly shows a dependence on microgel concentration. The compressibility of the core was very recently included in the treatment by Romeo and Pica Ciamarra ${ }^{22}$ who modified the inner potential using a different harmonic strength. We have also solved the $\mathrm{OZ}$ equation with RY closure for all these models in order to test whether they can be used to describe the structure of our microgel particles in the fluid regime.

\section{RESULTS AND DISCUSSION}

\section{A. Comparison of the measured $g(r)$ in 2 and 3 dimensions with theory/simulations}

Modern confocal laser scanning microscopes with their high scanning speed allow us to obtain reconstructed 3D images of a large number of colloidal particles in suspension, and thus offer direct access to quantities such as the pair correlation function $g(r)$. In the fluid state, the microgel particles are in a disordered state and constantly undergoing Brownian motion. When using the CLSM to probe a large ensemble of particles, the spatial resolution of the microscope which differs along the $\mathrm{z}$-direction and the $\mathrm{x}$-y image plane, its finite scanning speed and the exact nature of the dye distribution inside the particles significantly influence the determination of the particle coordinates. In turn this also significantly affects the quality of the obtained information on their microstructure such as the pair correlation function $g(r)$ calculated from this ensemble of particles. This results typically in a broadening of the peaks in $g(r)$, combined with a significant reduction of the peak height and a shift of their positions. These artifacts have already been noticed in previous studies with hard sphere-like ${ }^{30-32}$ and microgel colloids. ${ }^{24}$ Unfortunately, the extent of these various contributions in quantitative CLSM data varies with the system considered, and depends for example on $\phi_{\text {eff }}$ due to the concentration dependence of the particle mean square displacement $\left\langle\Delta r(t)^{2}\right\rangle$ in relation to the scanning speed, but also on the degree of particle labeling, the particle size and the mismatch of refractive index, to name some of the more important factors only. Any attempt to use CLSM data to determine $g(r)$ and subsequently gain insight on the pair potential between microgels at different packing fractions thus requires a careful analysis of these effects.

One way to improve the data quality obtained with fluid samples is to take into account the significant differences in resolution between the $\mathrm{z}$-axis and the $\mathrm{x}$-y-image plane, to avoid adding the $\mathrm{z}$-noise and to calculate a 2D-g(r) from thin optical slices instead. The differences between the thus obtained 3D- versus 2D- $g(r)$ data are illustrated in Fig. 2, where the corresponding pair correlation functions are shown for a sample with $\phi_{\text {eff }}=0.52$. We clearly see the significant differences between the two sets of data, with a much larger peak height for the 2D- $g(r)$, and a broader shoulder at small $r$ values for the $3 \mathrm{D}-g(r)$ that could be interpreted as the result of a much softer potential when relying on experimental 3D- $g(r)$ only.

However, in a real experimental situation it is difficult to achieve the very thin optical slices required for a true 2D- $g(r)$ due to the lower spatial resolution along the z-direction. As a consequence we will always see particles which have their center of mass away from the focal plane. That is, a CLSM picture generated by a standard $x-y$ scan will correspond to a thick illuminated sheet rather than an ideal 2D slice as shown

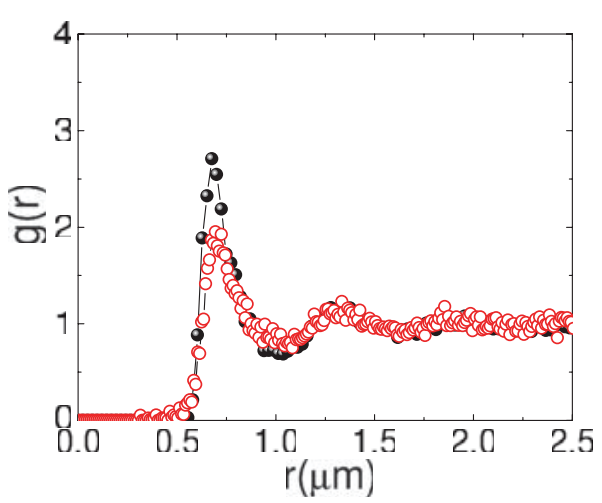

FIG. 2. A comparison of a 3D experimental $g(r)$ (red open symbols) at $\phi_{\text {eff }}$ $=0.52$ with the $2 \mathrm{D}-g(r)$ (black solid symbols) for the same sample. 
(a)
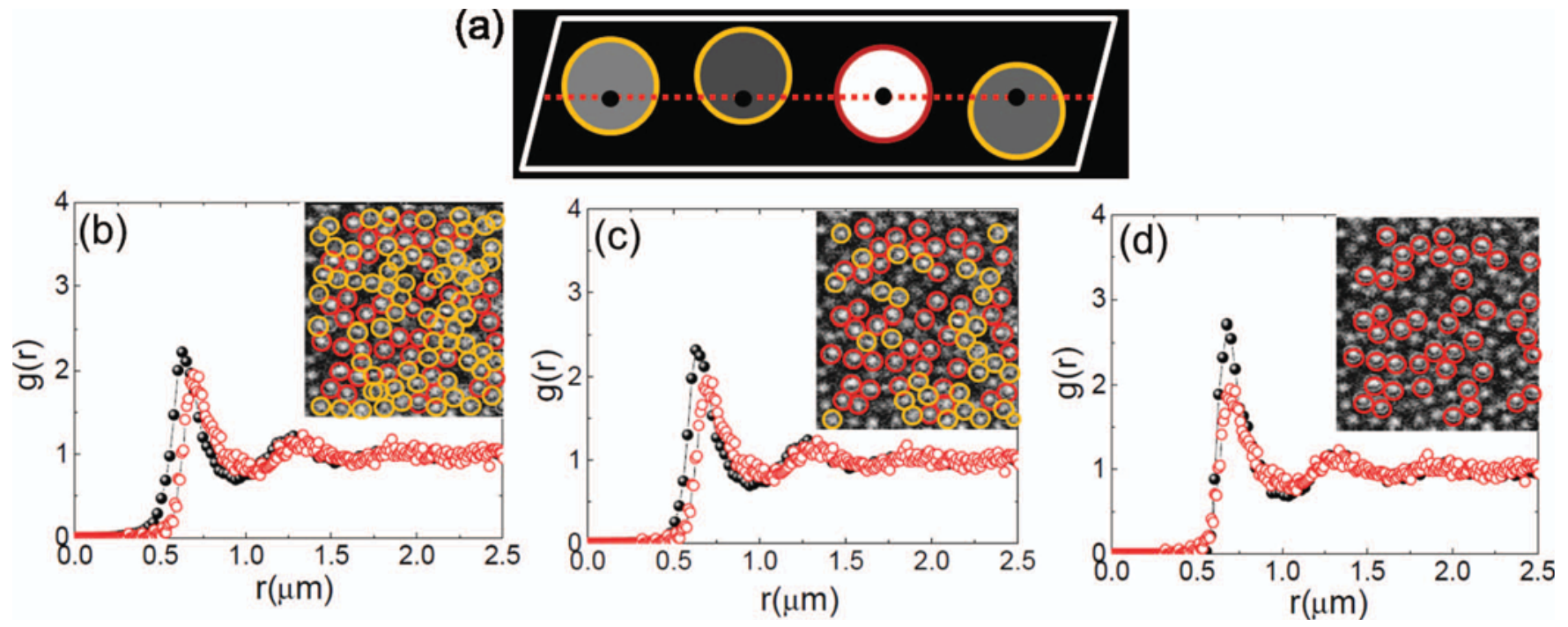

FIG. 3. (a) Schematic representation of the resolution problem encountered in CLSM studies of the microstructure present in a microgel dispersion. Crosssection of a x-y scan at constant $\mathrm{z}$ in the CLSM. The center of the bright particle (red line) is in the focal plane located in the center of the confocal volume, whereas the centers of all other less bright particles (yellow line) are located above and below the focal plane, thus creating a projection error in $2 \mathrm{D}$ and introducing a larger error in the in-plane $g(r)$. (b) The apparent 2D- $g(r)$ (black solid symbols) calculated using all particles seen in the X-y-scan is compared with that of a full 3D- $g(r)$ (open red symbols) at $\phi_{\text {eff }}=0.52$. Inset: Snapshot where the particles are labeled according to A based on their intensity (out of plane: yellow line; in-plane: red line). (c) Apparent 2D-g(r) (black solid symbols) where we have excluded the part of the out of focus particles that have the lowest intensity and are thus far away from the focal plane. The inset shows an example of a snapshot where the used particles are labeled either by a yellow (out of plane) or red (in plane) line. Also shown is the initial 3D- $g(r)$ (open red symbols). (D) "Correct" asymptotic 2D- $g(r)$ where only particles in the center plane are used. The inset shows an example of a snapshot where the used particles are labeled by the red line. Also shown is the initial $3 \mathrm{D}-g(r)$ (open red symbols).

in Fig. 3(a). This schematic figure demonstrates that the center of the bright particle with the red line is directly in the focal plane, while the center of the less bright particles marked with a yellow line are significantly away from the focal plane. This thus leads to a projection error in the in-plane 2D-g(r) at distances comparable to the thickness of the slice. As a result, the thus obtained 2D- $g(r)$ from untreated individual 2D images will not be identical with an "ideal" 3D- $g(r)$, as pointed out by previous studies. ${ }^{2}$ This problem has been overcome by discriminating particles which are far from the focal plane based on their intensity. Such a post-treatment of CLSM images allows us to create a correct 2D image of particles that have their center in a chosen plane and to calculate a correct $2 \mathrm{D}-g(r)$ that now is equivalent to an ideal $3 \mathrm{D}-g(r)$ in the absence of all resolution effects and projection errors. We have adopted this method and obtain 2D- $g(r)$ from thin slices as illustrated in Figs. 3(b)-3(d) for $\phi_{\text {eff }}=0.52$. Here we systematically remove all particles from the $2 \mathrm{D}$ images that are not in the plane centered at the middle of the CLSM focus, and accordingly reduce the noise normally introduced by the contributions from out of focus particles, with a significant effect on the resulting $2 \mathrm{D}-g(r)$.

Fig. 3(b) demonstrates the dramatic effect from the projection error on the resulting apparent 2D-g(r) when we use all particles seen in a $\mathrm{x}-\mathrm{y}$ scan. As a result, the thus obtained $2 \mathrm{D}-g(r)$ is significantly broadened at low $r$-values, and has a reduced peak height. Moreover, the first peak of the 2D$g(r)$ is shifted when compared to the 3D- $g(r)$. Figs. 3(c) and $3(d)$ show the evolution of the 2D- $g(r)$ when we start to remove out of focus particles. We finally obtain a fully corrected asymptotic 2D- $g(r)$ for a given sample after collecting 4000-
5000 frames from $x-y$ scans in different positions in the bulk suspension, and analyzing these images using standard methods to obtain ensemble-averaged pair correlation functions. ${ }^{33}$ The corresponding 2D- and 3D- $g(r)$ differ as expected in peak width and height, but the peak positions are almost overlapping. These pair correlation functions now provide the basis for a comparison with computer simulations in order to assess the effective interaction potential between the microgels at different effective volume fractions.

However, it is clear that such procedures are not always unambiguous, and we thus perform additional systematic computer simulations of the corresponding experimental conditions in order to demonstrate the consistency of the experimentally obtained 2D- and 3D- $g(r) s$. Here we simulate the effect of the projection error on the calculation of the 2D$g(r)$ 's analogous to the situation sketched in Fig. 3(a), and the effect of the lower z-resolution and thus the added noise on the experimental z-coordinates in the calculation of the 3D$g(r)$ from the experimental data for $\phi_{\text {eff }}=0.52$. The results from these simulations are summarized in Figs. 4(a) and 4(b), respectively. In the simulations, we use particles with a diameter $\sigma=0.714 \mu \mathrm{m}$ and a polydispersity of $4 \%$, interacting via a soft Hertzian potential given by Eq. (4) with a potential strength $\epsilon=496 k_{B} T .^{17}$

In Fig. 4(a), we see that with increasing slice thickness, the corresponding 2D-g(r) systematically deviates from the true 3D- $g(r)$ with respect to peak position and peak height. The 2D- $g(r)$ also shows a broadening at low $r$ with increasing slice thickness. The simulations thus reproduce the experimental observations described in Fig. 3. It is however important to point out that a slice thickness of half the 

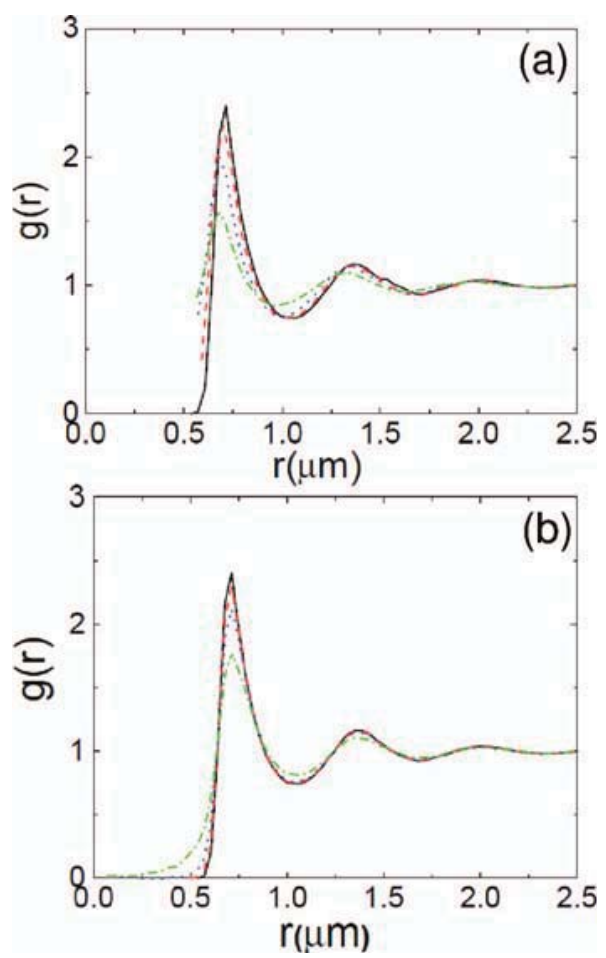

FIG. 4. (a) Simulated 2D-g(r)'s for different slice thicknesses (in units of the particle diameter $\sigma$ ) in comparison to the simulated 3D (black solid line) $g(r)$ at $\phi_{\text {eff }}=0.52$. Red dashed, blue dotted, and green dashed dotted lines correspond to slice thickness $0.5 \sigma, 1.0 \sigma$, and $2.0 \sigma$, respectively. (b) Simulated 3D- $g(r)$ obtained with different z-noise values at the same $\phi_{\text {eff }}=0.52$. Black solid, Red dashed, blue dotted, and green dashed dotted lines correspond to z-noise $0 \%, 1.0 \%, 2.0 \%$, and $5.0 \%$, respectively. See text for more details.

diameter $\sigma$, i.e., a condition that can easily be matched by our ability to use the intensity as a measure of the particle position within the confocal volume, is virtually identical with the correct 3D- $g(r)$. This confirms that our experimentally determined 2D-g(r) correctly reproduces the 3D structure of the suspensions. The simulations with increasing added z-noise shown in Fig. 4(b) demonstrate that the peak height in $g(r)$ decreases and the broadening at lower $r$ increases with increasing z-noise, while the peak position remains unchanged.

We now test the consistency of the experimentally determined 2D- and 3D- $g(r)$ pair correlation functions. This is demonstrated in Fig. 5, where we compare both experimental data sets with the corresponding 3D- $g(r)$ obtained from simulations and the Rogers-Young (RY) calculation. Fig. 5 shows that both data sets agree very well with the predictions for a Hertzian potential with $\epsilon=496 k_{B} T$ when taking into account an additional $\mathrm{z}$-noise of $2.5 \%$ in the simulation of the 3D- $g(r)$ (Fig. 5(a)), confirming the consistency of our approach to experimentally determine the correct liquid structure.

\section{B. Density dependence of $g(r)$ in the fluid phase}

The findings summarized in Fig. 5 allow us to draw a few important first conclusions. The pair correlation functions $g(r)$ derived from theory and simulations using a model of particles interacting via a Hertzian interaction potential with
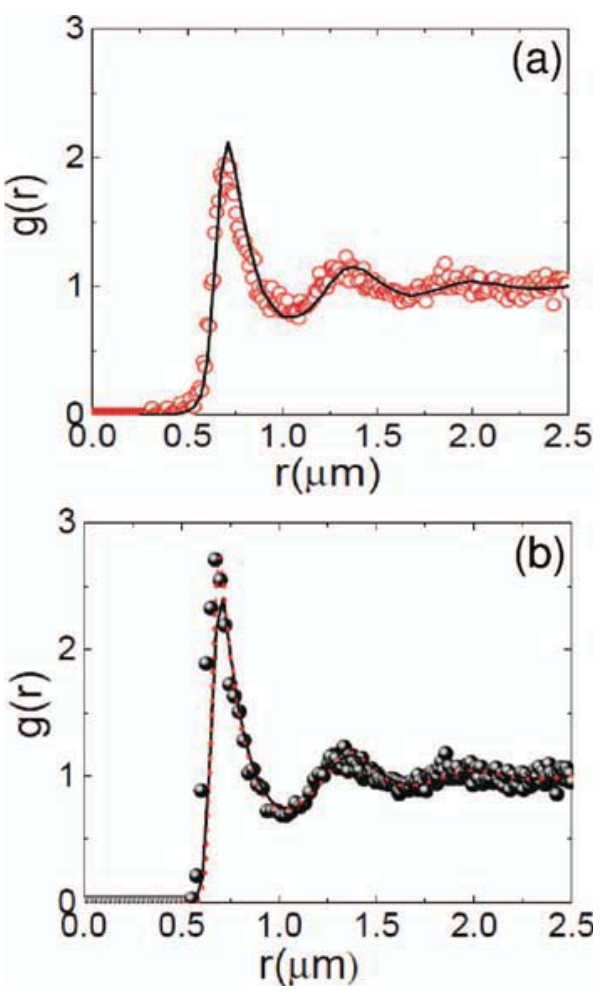

FIG. 5. (a) Experimental 3D-g(r)'s (open red symbols) compared with the $3 \mathrm{D}-g(r)$ obtained from a simulation with added z-noise of $2.5 \%$ at $\phi_{\text {eff }}=$ 0.52 (b) Experimental 2D- $g(r)$ (solid black symbols) from a thin 2D slice compared with the corresponding 3D- $g(r)$ from theory (dotted red line) and simulation(solid black line) with no further added z-noise at $\phi_{\text {eff }}=0.52$. Simulations are done with $4 \%$ of polydispersity. See text for details.

$\epsilon=496 k_{B} T$ are in very good agreement with the experimental $g(r)$ in 3- and 2-dimensions at $\phi_{\text {eff }}=0.52$, provided that the lower resolution of the CLSM in the z-direction is taken into account for the analysis of the 3D data. The 2D-g(r) from a properly constructed thin slice by discriminating the out of focus particles is virtually noise-free, and is also suffering less from the additional noise caused by the Brownian motion of the particles. In the following, we thus concentrate on calculating 2D-g(r), where we first construct thin slices from $x-y$-scans, and study structural ordering as a function of $\phi_{\text {eff }}$ throughout the fluid regime.

Fig. 6 shows the experimentally measured 2D-g(r) at different $\phi_{\text {eff }}$. At the lowest $\phi_{\text {eff }}=0.154$, where particle positions can still be measured with sufficient accuracy, $g(r)$ has a weak first peak and quickly approaches its asymptotic value of one at higher $r$ and thus exhibits the typical features of a weakly correlated liquid state. With increasing $\phi_{\text {eff }}, g(r)$ shows a systematic increase in the height of the first peak, and higher order peaks also develop due to the increasing spatial correlations among the particles as the density increases. The experimental results at different $\phi_{\text {eff }}$ are then compared with the theoretical predictions for Hertzian spheres obtained from RY and simulations, shown respectively in Figs. 6(a) and 6(b). In both cases we keep the strength of the repulsion and diameter fixed and only vary $\phi_{\text {eff }}$. The good agreement between experimental and theoretical data for all densities investigated, strongly supports a model where the microgel size and thus 

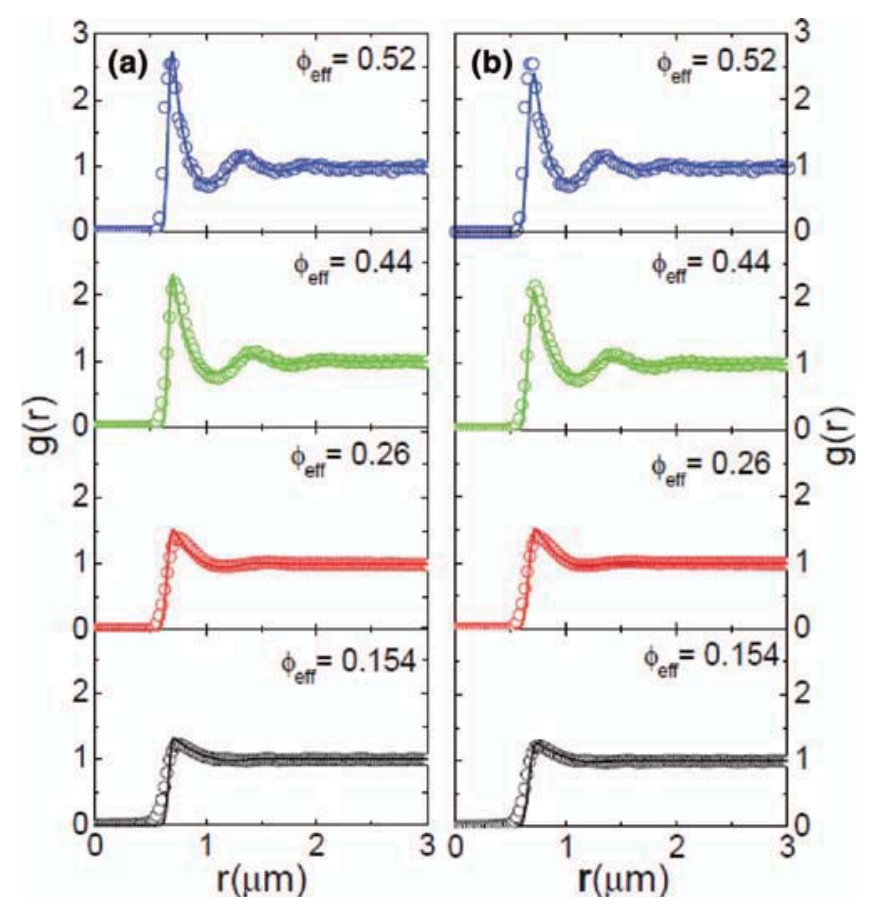

FIG. 6. Experimentally measured 2D pair-correlation functions 2D- $g(r)$ (open circles) at different $\phi_{\text {eff }}$ at a temperature $T=15^{\circ} \mathrm{C}$. Lines represent $g(r)$ obtained from (a) theory (RY) and (b) from simulation for the same $\phi_{\text {eff }}$ as in the experiments and for a fixed diameter $\sigma=0.714 \mu \mathrm{m}$ and a strength of the Hertzian repulsion of $\epsilon=496 k_{B} T$. Simulations are done with $4 \%$ of polydispersity.

also the interaction potential remains independent of concentration throughout the fluid domain, i.e., almost up to close packing. The simulations with added polydispersity are in slightly better agreement with experiments than the monodisperse RY, but the differences are minor except at the highest volume fraction, which is not surprising given the highly monodisperse nature of the particles and the very soft potential. For the lowest concentrations investigated $\phi_{\text {eff }}=0.154$ and 0.26 , the first peak of $g(r)$ appears to be slightly broadened and the correlation function increases less steeply than predicted at small distances $r<\sigma$. We believe that this is mostly due to the enhanced diffusivity of the particles at low values of $\phi_{\text {eff }}$ during the duration of a scan and a reduced statistical accuracy given the significantly reduced total number of particles sampled. We estimate this additional error caused by the Brownian motion of the particles to add an uncertainty of approximately $1 \%$ for the $x-y$ positions in case of unrestricted free particle diffusion at lower $\phi_{\text {eff }}$, and less in case of nearly arrested particle motion at higher $\phi_{\text {eff }}$ close to the glass transition. In addition, at lower $\phi_{\text {eff }}$ particle tracking errors can also contribute to some extent.

Finally, we make some general remarks on the quality of the agreement of typical experimental $g(r)$ 's obtained for colloidal suspensions deduced from real space studies using fluorescent confocal microscope with those obtained from theory/simulations. Minor but noticeable discrepancies between model (simulation) and experimental results have also been observed in previous studies on well-defined classical hard sphere systems ${ }^{31,34}$ or hard sphere colloids with depletion interactions. ${ }^{30}$ We feel that the most important point for claiming quantitative agreement is the ability to not only reproduce an individual $g(r)$, but recover the volume fraction dependence of the main features of $g(r)$. In our case, we clearly demonstrate that once we fix the strength of the Hertzian interaction parameter $\epsilon$ for one concentration, we can reproduce the experimental data within experimental accuracy over a fairly extended range of densities without any additional adjustable parameters.

\section{Testing different potential models}

We now solve the $\mathrm{OZ}$ equation for harmonic and inverse power law potentials with $n=6,12$ for the fluid packing fractions whose $g(r)$ have been measured via CLSM, namely, $\phi=0.154,0.26,0.44,0.52$, respectively. The repulsive strength of each model has been chosen to match the peak height of $g(r)$ for the Hertzian at $\phi=0.154$. We then compare the resulting $g(r)$ 's with the results obtained with the Hertzian potential which match the experimental data within experimental errors. Although the OZ-RY results are approximate, we build on the fact that RY works quite well for the Hertzian potential in the considered range of densities, therefore we expect that it will work reasonably well also for other potentials in the studied fluid regime. From this analysis, we thus can assess whether the correct density dependence of $g(r)$ is also captured by the different potentials.

In Fig. 7(a), the various potentials are shown. The resulting $g(r)$ 's are reported in Fig. 7(b). Here, in the left panel, Hertzian and harmonic results are compared. It is easy to notice that the results are virtually indistinguishable in the range of densities investigated. A real distinction between them could become more evident at larger packing fractions, i.e., beyond the fluid regime, and will be addressed in future work. For the present study, we thus conclude that we could equally adopt either of the two models in order to describe the structure of our microgel suspensions in the entire fluid regime. However, our preference goes to the Hertzian model because it correctly describes the interactions between elastic spheres in a regime of not-too-large compression, hence the regime under investigation in this study.

Contrarily to the Hertzian and harmonic models, the family of inverse power law potentials for the softness parameters commonly used in the literature fails to capture the behavior of the experimental $g(r)$ in the investigated fluid region. Indeed, as shown in the right panel of Fig. 7, we find that both studied cases $(n=6,12)$, once we have fixed the repulsion strength $\epsilon_{n}$, display a much stronger variation of the peak position (even when taking into account a rescaled core distance due to the softness): while the Hertzian and harmonic potentials result in a variation that is of the order of $1 \%-2 \%$ in the investigated range of densities, for $n=6$ the peak position decreases by around $25 \%$ between the two extreme packing fractions and for $n=12$ the variation reaches $15 \%$. In addition, the peak heights are respectively lower (higher) than the Hertzian/experimental ones with increasing density for $n$ $=6(n=12)$. Therefore, in order to match the experimental results one would need to adjust either the repulsive strength or the effective diameter in Eq. (6) at each density (with a considerable variation throughout the studied state points). 

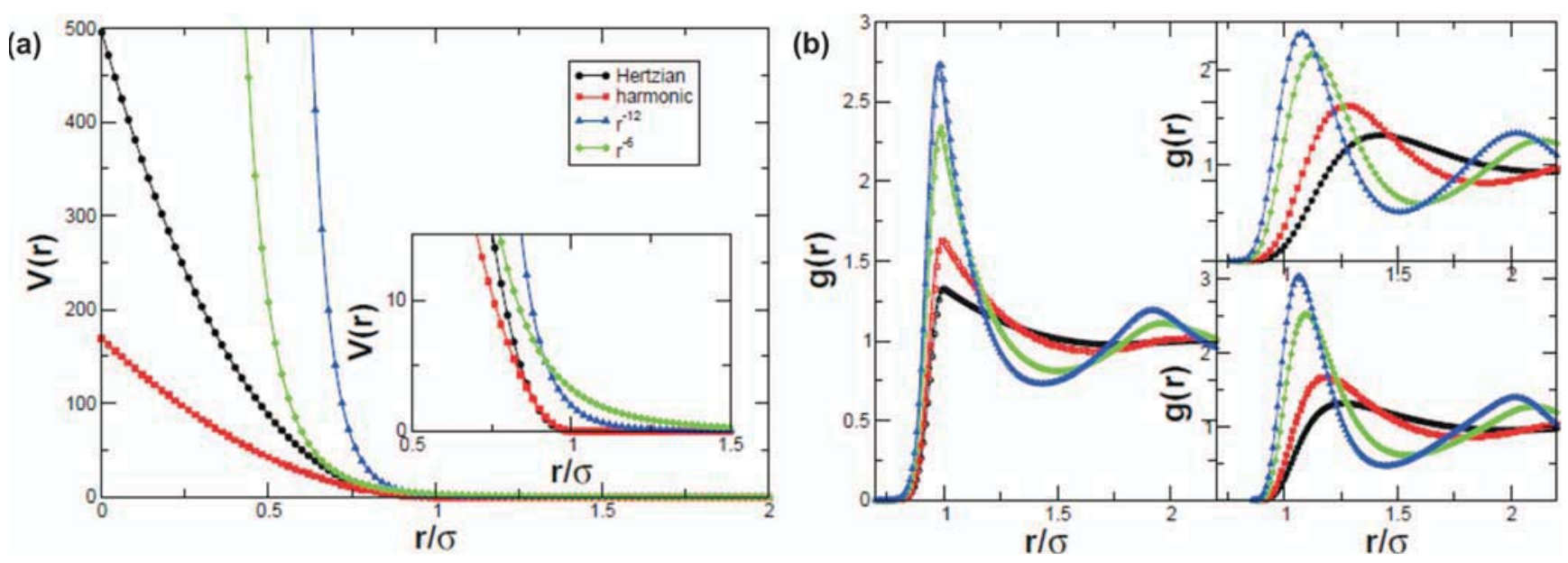

FIG. 7. (a) Different potentials used. Their strengths have been chosen to match the peak height of $g(r)$ for the Hertzian at $\phi=0.154$. Hence: $\epsilon_{\text {harmonic }}$ $=169 k_{B} T, \epsilon_{6}=3.25 k_{B} T$ and $\epsilon_{12}=1.95 k_{B} T$. (b) Radial distribution functions for the investigated packing fractions $\phi=0.154,0.26,0.44,0.52$ for the potentials described above. On the left panel harmonic and Hertzian predictions are superimposed and virtually indistinguishable; on the right the inverse power law results for $n=6$ (top) and $n=12$ (bottom) are reported.

This is at odds with independent neutron scattering experiments $^{35}$ that unambiguously demonstrate that the size and shape of the particles remain constant throughout the fluid regime. Therefore, we conclude that inverse power law potentials have to be discarded in terms of a description of the structure of our microgel particle suspensions in the fluid regime.

We now examine the brush-like model proposed in Ref. 15. We start by considering the harmonic model for the corona layer with varying spring constant as described by the Alexander-De Gennes model throughout the whole range, hence in conditions of a fully compressible core. Then, we compare this to the case where the harmonic corona is complemented by an incompressible core modeled by a $r^{-36}$ strong repulsion. Both studies use the experimental parameters for the inner core $(0.76 \sigma)$ and the corona layer (width $0.24 \sigma)$. We use the same repulsion strength at the lowest density of $\phi=0.154$ as for the purely harmonic potential stud-

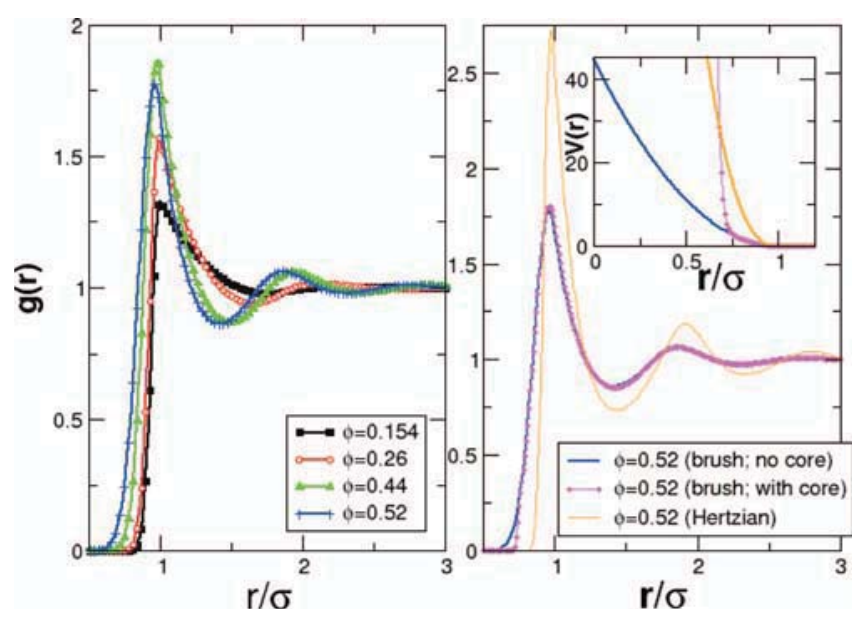

FIG. 8. (Left) RY predictions for the $g(r)$ at $\phi=0.154,0.26,0.44,0.52$ for the brush model introduced in Ref. 15 without an incompressible core; (Right) $g(r)$ for $\phi=0.52$ for the brush with a fully compressible core and one with an incompressible core, compared with the corresponding Hertzian results. In the inset, we compare the brush potentials with compressible/incompressible core for $\phi=0.52$ with the Hertzian model. ied above. With increasing density, the strength of repulsion decreases from $169 k_{B} T$ to $41 k_{B} T$ at $\phi=0.52$, and the resulting $g(r)^{\prime} s$ do not reproduce the experimentally observed density dependence as can be seen in Fig. 8. When we add an incompressible core, we notice that for the parameters under consideration, this has little effect on the $g(r)$ because at the inner core, the repulsive barrier is already quite high $\sim 5-10 k_{B} T$ for the studied range of strengths. The only noticeable effect is the sharp decrease of $g(r)$ at the inner core. We expect that the role of the core might become more and more dominant either for higher densities or for smaller strengths, where particles would be more easily allowed to overlap.

\section{CONCLUSIONS}

We have shown that the microstructure in suspensions of swollen microgel particles as expressed through the pair correlation function $g(r)$ is in very good agreement with a model of particles interacting through a soft Hertzian potential with fixed repulsion strength. No evidence was found for a concentration dependence of the particle diameter and the strength of the interaction throughout the fluid regime, i.e., for $\phi_{\text {eff }}<\phi_{\text {freezing. }}$. While confocal laser scanning microscopy is an ideal tool to directly determine particle coordinates and thus $g(r)$, care has to be taken to avoid systematic errors in $g(r)$ due to the reduced resolution of the CLSM in the $z$-direction. Here simulations compared with a quantitative analysis of the deviations of the $z$-coordinate of the particles imaged in fast $x-y$-scans based on their fluorescent intensity allow us to either include the resolution in the analysis of experimental 3D- $g(r)$ 's, or experimentally determine virtually noise-free 2D- $g(r)$ 's. Our study thus for the first time provides a set of structural data over an extended range of concentrations that together with simulations allows us to develop a consistent description of the microstructure based on a Hertzian-type interaction potential and using a particle size that has been independently determined from DLS and SAXS. It will of 
course be interesting now to extend these investigations to higher densities above close packing, where previous studies have described intriguing dynamic behavior, and where we expect that the particle size and interaction potential may eventually respond to the ultra-high density.

\section{ACKNOWLEDGMENTS}

This work was supported by the Swiss National Science Foundation, the Swedish Research Council (Project No. 621-2011-4338) and the science faculty of Lund University. E.Z. acknowledges support from MIUR-FIRB ANISOFT (RBFR125H0M) and MIUR-PRIN. SAXS experiments were performed at the cSAXS beam line of the Swiss Light Source at the Paul Scherrer Institute, Switzerland, and we gratefully acknowledge help from our local contact Dr. Andreas Menzel. We are grateful to Dr. Urs Gasser for the determination of the CLSM resolution.

${ }^{1}$ P. N. Pusey and W. van Megen, Nature (London) 320, 340 (1986).

${ }^{2}$ W. K. Kegel and A. van Blaaderen, Science 287, 290 (2000).

${ }^{3}$ E. R. Weeks, J. C. Crocker, A. C. Levitt, A. B. Schofield, and D. A. Weitz, Science 287, 627 (2000).

${ }^{4}$ U. Gasser, E. R. Weeks, A. Schofield, P. N. Pusey, and D. A. Weitz, Science 292, 258 (2001).

${ }^{5}$ C. N. Likos, H. Löwen, M. Watzlawek, B. Abbas, O. Jucknischke, J. Allgaier, and D. Richter, Phys. Rev. Lett. 80, 4450 (1998).

${ }^{6}$ D. M. Heyes and A. C. Branka, Soft Matter 5, 2681 (2009).

${ }^{7}$ L. Berthier, A. J. Moreno, and G. Szamel, Phys. Rev. E 82, 060501(R) (2010).

${ }^{8}$ P. S. Mohanty and W. Richtering, J. Phys. Chem. B 112, 14692 (2008).

${ }^{9}$ J. Santos, B. Sierra-Martin, R. Vavrin, Z. Hu, U. Gasser, and A. FernandezNieves, Macromolecules 42, 6225 (2009).

${ }^{10}$ H. Senff and W. Richtering, J. Chem. Phys. 111, 1705 (1999).

${ }^{11}$ M. Stieger, W. Richtering, J. S. Pedersen, and P. Lindner, J. Chem. Phys. 120, 6197 (2004).

${ }^{12}$ M. Das, H. Zhang, and E. Kumacheva, Annu. Rev. Mater. Res. 36, 117 (2006).
${ }^{13}$ D. A. Sessoms, I. Bischofberger, L. Cipelletti, and V. Trappe, Philos. Trans. R. Soc. A 367, 5013 (2009).

${ }^{14}$ Z. Meng, J. K. Cho, V. Breedveld, and L. A. Lyon, J. Phys. Chem. B 113, 4590 (2009).

${ }^{15}$ F. Scheffold, P. Diaz-Leyva, M. Reufer, N. B. Braham, I. Lynch, and J. L. Harden, Phys. Rev. Lett. 104, 128304 (2010).

${ }^{16}$ Z. Zhang, N. Xu, D. T. N. Chen, P. Yunker, A. M. Alsayed, K. B. Aptowicz, P. Habdas, A. J. Liu, S. R. Nagel, and A. G. Yodh, Nature (London) 459, 230 (2009).

${ }^{17}$ D. Paloli, P. S. Mohanty, J. J. Crassous, E. Zaccarelli, and P. Schurtenberger, Soft Matter 9, 3000 (2013).

${ }^{18}$ M. Laurati, J. Stellbrink, R. Lund, L. Willner, E. Zaccarelli, and D. Richter, Phys. Rev. E 76, 041503 (2007).

${ }^{19}$ S. Prestipino, F. Saija, and P. V. Giaquinta, J. Chem. Phys. 123, 144110 (2005).

${ }^{20}$ J. Pamies, A. Cacciuto, and D. Frenkel, J. Chem. Phys. 131, 044514 (2009).

${ }^{21}$ Y.-L. Zhu and Z.-Y. Lu, J. Chem. Phys. 134, 044903 (2011).

${ }^{22}$ G. Romeo and M. P. Ciamarra, Soft Matter 9, 5401 (2013).

${ }^{23}$ M. Stieger, J. S. Pedersen, P. Lindner, and W. Richtering, Langmuir 20, 7283 (2004).

${ }^{24}$ J. K. Cho, Z. Meng, L. A. Lyon, and V. Breedveld, Soft Matter 5, 3599 (2009).

${ }^{25}$ J. R. Seth, R. T. Bonnecaze, and M. Cloitre, J. Rheol. 50, 353 (2006).

${ }^{26}$ J. P. Hansen and I. R. McDonald, Theory of Simple Liquids, 3rd ed. (Academic Press, New York, 2006).

${ }^{27}$ A. Lang, G. Kahl, C. N. Likos, H. Löwen, and M. Watzlawek, J. Phys. Condens. Matter 11, 10143 (1999).

${ }^{28}$ P. Kumar, S. V. Buldyrev, F. Sciortino, E. Zaccarelli, and H. E. Stanley, Phys. Rev. E 72, 021501 (2005).

${ }^{29}$ C. Mayer, E. Stiakakis, E. Zaccarelli, C. N. Likos, F. Sciortino, P. Tartaglia, H. Löwen, and D. Vlassopoulos, Rheol. Acta 46, 611 (2007).

${ }^{30}$ C. P. Royall, A. A. Louis, and H. Tanaka, J. Chem. Phys. 127, 044507 (2007).

${ }^{31}$ R. P. A. Dullens, D. G. A. L. Aarts, and W. K. Kegel, Proc. Natl. Acad. Sci. 103, 529 (2006).

${ }^{32}$ C. P. Royall, M. E. Leunissen, A.-P. Hynninen, M. Dijkstra, and A. van Blaaderen, J. Chem. Phys. 124, 244706 (2006).

${ }^{33}$ J. C. Crocker and D. G. Grier, J. Colloid Interface Sci. 179, 298 (1996).

${ }^{34}$ M. Brunner, C. Bechinger, U. Herz, and H. von Grünberg, Europhys. Lett. 63, 791 (2003).

${ }^{35}$ P. S. Mohanty, S. Nöjd, M. Obiols-Rabasa, J. Crassous, K. van Gruijthuijsen, A. Stradner, and P. Schurtenberger, "Escaping the squeeze: Soft particles at ultrahigh densities" (unpublished). 